\title{
Potential role of cellular senescence on coronavirus infections
}

\author{
Prem Chapagain ${ }^{1,2 *}$ \\ ${ }^{1}$ Department of Physics, Florida International University, Miami, FL 33199, USA \\ ${ }^{2}$ Biomolecular Sciences Institute, Florida International University, Miami, FL 33199, \\ *E-mail: chapagap@fiu.edu
}

\begin{abstract}
The disproportionate incidences of COVID-19-related hospitalization and mortality for different age groups and various underlying health conditions is a result of a complex social predisposition to the exposure, resistance, and tolerance for the infection. Based on the observed data as well as the molecular mechanisms for viral entry and replication, cellular senescence related to aging, obesity, hypertension, and diabetes appears to be strongly correlated with the SARS-CoV-2 infections resulting in higher COVID-19 related complications and mortality. Establishing such a correlation may allow us to better explain the pathobiology as well as the differential nature of the SARS-CoV-2 infections and consider targeted control and therapeutic strategies to combat the disease.
\end{abstract}

Keywords: cellular senescence; SARS-CoV-2; coronavirus; aging

\section{Introduction}

It has become abundantly clear that older adults and individuals with underlying health conditions such as diabetes, obesity, or heart problems, or smoking are at most risk of serious complications or death due to COVID-19 ${ }^{1}$. A recent population-based study by the Center for Disease Control and Prevention (CDC) using the surveillance network COVID-NET showed that nearly $75 \%$ of all patients hospitalized for COVID-19 during a 4-week period in March 2020 were aged $>50$ years ${ }^{2}$. Similarly, hospitalization data suggested that males and black population are disproportionately affected by the disease. Among younger adult (18-64 years) patients, obesity was the most prevalent underlying condition, followed by hypertension, diabetes, and asthma ${ }^{2}$. The disproportionate incidences of SARS-CoV-2 infections in different age group, race, or gender is a result of a complex social predisposition to the exposure, resistance, and tolerance for the 
infection. Understanding the molecular mechanisms of how SARS-CoV-2 differentially infects individuals will allow targeted control and therapeutic strategies to combat the disease. So, why does COVID-19 disproportionately affect the elderly, obese, and diabetic demographics? Based on the observed data as well as the molecular mechanisms for viral entry and replication, cellular senescence related to aging, obesity, and diabetes appears to be strongly correlated with the SARSCoV-2 infections resulting in higher COVID-19 incidences of infections and mortality (Box 1). This means that these viruses are possibly exploiting the very mechanism intended for eliciting the immune response for senescent cell removal. Very recently, Malavolta et al. ${ }^{3}$ also surveyed therapeutic molecules targeting senescent cells drawing relevance to COVID-19. This brief review aims to stimulate the discussion on the potential role of cellular senescence in COVID-19 as well as other viral infections. Recent reports suggest that some SARS-CoV-2 infected individuals can harbor the virus for months. The long incubation time and persistence in the body may also be related to cellular senescence as it is shown that viruses can remain infections for weeks in senescent cells ${ }^{4}$.

Box 1: Correlations of cellular senescence and various underlying conditions implicated in COVID-19. Many of these conditions and senescence may have feedback to one another and exacerbate the effects.

\begin{tabular}{|l|l|l|}
\hline Factor & $\begin{array}{l}\text { Cellular } \\
\text { Senescence }\end{array}$ & $\begin{array}{l}\text { COVID-19 } \\
\text { incidence }\end{array}$ \\
\hline Age & $\uparrow$ & $\uparrow$ \\
\hline Obesity & $\uparrow$ & $\uparrow$ \\
\hline Type 2 Diabetes & $\uparrow$ & $\uparrow$ \\
\hline Cigarette Smoking & $\uparrow$ & $\uparrow$ \\
\hline Hypertension & $\uparrow$ & $\uparrow$ \\
\hline
\end{tabular}

\section{Cellular senescence}

Cellular senescence is a process of irreversible cell-cycle arrest that renders previously replication-competent cells non-proliferative but still metabolically active ${ }^{5}$. A number of factors such as DNA damage due to oxidative or chemotherapy-induced stress, mitochondrial dysfunction, 
telomere erosion contribute to cellular senescence ${ }^{6}$. Normal cells are equipped with repair mechanisms and the process may include temporary growth arrest. If the repair fails, damaged cells may either initiate regulated cell death or become senescent. In general, it is a regulated response to stress and plays important roles in several essential physiological processes, from regulating bone growth during childhood to wound healing or tissue regeneration after injury and suppressing tumor progression (see reviews ${ }^{7,8}$ ). Key features of senescent cells include damage sensing signaling pathways activation and expression of anti-proliferative molecules resulting in durable growth arrest. While cellular senescence has important functions, the accumulation of senescent cells with age is implicated in a number of age-associated conditions, including diabetes ${ }^{9}$ as well as cardiovascular ${ }^{10}$ and pulmonary diseases ${ }^{11}$. The increase in stress-induced cellular senescence is a result of cumulative cell damage with age and failure of the immune system to eliminate them ${ }^{12}$. In addition to aging, obesity also leads to an increase in senescent cells ${ }^{13-15}$. Fat tissues acquired from patients with diabetic tissues show characteristics of senescent-like state with elevated expression of p53 and p21, suggesting that diabetes is associated with cellular senescence in fat tissue ${ }^{16}$. Similarly, hypertension is shown to cause cellular senescence ${ }^{16,17}$. Conditions such as age, obesity, diabetes, and hypertension are, therefore, inter-related and associated with cellular senescence. Interestingly, all of these conditions are increased risk factors for COVID-19 complications. In addition, one of the primary markers of cellular senescence is leukocyte telomere shortening ${ }^{18}$, which seems to occur at a differential rate among different demographics, with a higher rate among men and the black population ${ }^{19,20}$.

Senescent cells are tagged with markers that can be recognized by the immune system for elimination ${ }^{21}$. They express factors such as MHC class I polypeptide-related sequence A (MICA) and UL16 binding protein 2 (ULBP2) which serve as the ligands to bind to the natural killer (NK) cell receptor NKG2D, thereby activating the cell removal ${ }^{22,23}$. This process plays a central role in tissue remodeling during carcinogenesis and tumor progression. In this respect, senescent cells don't just block the cell proliferation but also prime themselves for elimination ${ }^{24}$. They also secrete a plethora of pro-inflammatory cytokines, chemokines, and growth factors, grouped as senescenceassociated secretory phenotype (SASP) ${ }^{25}$. For example, the accumulation of tumor suppressor molecules $\mathrm{p} 16^{\mathrm{INK} 4 \mathrm{a}}$ and P53 are key markers of senescent cells.

In addition to the NK cell and macrophages, other innate and adaptive immune systems are also suggested to play a role in senescent cell surveillance. For example, the adaptive response to 
oncogene-induced senescent cells involves T cells (CD4) and MHC class II molecules on the cell surface ${ }^{26,27}$. Similarly, IgM antibodies are able to recognize senescent cells by binding to surfaceexposed molecules, including an oxidized form of vimentin filament presented on the outer surface of senescent cells ${ }^{28}$, suggesting the availability of a variety of new antigens presented on the senescent cell surface for immune system recognition and elimination ${ }^{24}$. Therefore, reduction in immune clearance in immunosuppressive conditions due to aging results in the accumulation of senescent cells.

\section{Viral infections and the role of cellular senescence}

Cellular senescence appears to play a dual or differential role in infections by different viruses. While cellular senescence is implied in impaired viral infection and is considered to be an important protection mechanism against oncogenic virus effects ${ }^{29,30}$, it is also suggested to enhance viral replication ${ }^{31}$. Persistent viral or oncogenic stresses preferentially induce senescence in normal cells. In response to viral infection, cells produce a high concentration of $\alpha$ and $\beta$ interferon cytokines localized in the sites of infection ${ }^{32}$. It is shown that normal cells can turn into senescent cells upon prolonged treatment with $\beta$-interferon leading to activation of DNA-damage signaling and p53 tumor suppressor ${ }^{33}$. Cytokines secreted by the infection-induced senescent cells can further encourage senescence in normal neighboring cells thereby stimulating immune response ${ }^{34}$ and potentially conferring protection from infection. It has been shown that senescent cells are resistant to vesicular stomatitis virus (VSV) infection and replication in vivo ${ }^{29}$, likely due to the recruitment of the immune system mediated by SASP molecules. Several viruses, including Hepatitis B virus (HBV) X ${ }^{35}$, Simian virus-40 (SV40) ${ }^{36}$, and Human Papillomaviruses (HPV) ${ }^{37}$ have developed mechanisms to either bypass or defeat cellular senescence for efficient viral replication. However, the role of cellular senescence appears to be more complicated. For example, it is found that the influenza virus (IFV) and the Varicella Zoster virus (VZV) replicate more efficiently in senescent cells than normal cells due to the incompetence of senescent cells in controlling the replication ${ }^{31}$. In addition, either knockdown of anti-senescent gene sirtuin 1 (SIRT1) in normal primary human epithelial and fibroblast cells, or the inhibition of SIRT1 activities by its inhibitor nicotinamide, enhanced viral replication, providing further evidence that senescent cells lacking SIRT1 can accelerate IFV replication ${ }^{31}$. 


\section{Potential Role of senescence on molecular mechanisms of coronavirus infections}

Although our understanding of how SARS-CoV-2 exploits cellular senescence is still lacking, a multitude of studies on the coronavirus entry as well as the features of the senescent cells provide a strong ground that cellular senescence plays an indispensable role in virus particle attachment on the host cell surface and infection. A few specifics that connect the related viral infections to cellular senescence and their relevance to SARS-CoV-2 are given below.

Efficient SARS-CoV and Enterovirus 71 (EV71) infections require surface Vimentin, which is a key component of the senescent cell surface.

It has recently been shown that senescent lung fibroblasts express and expose an oxidized form of membrane-bound vimentin on their cell surface ${ }^{28}$, and therefore the cell-surface vimentin is a novel marker for senescent fibroblasts. The ability of the $\operatorname{IgM}$ isotype of the innate immune system to recognize and bind the cell surface vimentin suggests that the oxidized membrane-bound vimentin is likely presented on the surface by the senescent cells for their removal by the immune system. Failure to remove senescent cells due to impaired immune system results in the accumulation of these vimentin-rich senescent cell surfaces, and some viruses, including SARS$\mathrm{CoV}$ and Enterovirus 71 (EV71) have found ways to exploit this.

SARS-CoV and SARS-CoV-2 spike proteins utilize the angiotensin-converting enzyme 2 (ACE2) as the receptor to latch on host cell surface. Other receptors such as the C-type lectin (CD209L) also facilitate the viral entry ${ }^{38}$. A recent study showed that the formation of spike protein-ACE2 is not sufficient for the SARS-CoV entry to infect the host cells and that surface vimentin is also required ${ }^{39}$. This study suggested that the cell surface vimentin directly interacts with the SARS-CoV spike protein during viral entry. Along with SARS-CoV, surface vimentin has also been shown to be an attachment receptor for EV71 ${ }^{40}$ as well as for endothelial infection by Cowpea mosaic virus (CPMV) ${ }^{41}$. Cells pretreated with vimentin fragments resulted in lower virus yields as it competitively inhibited the EV71 infection ${ }^{40}$. Based on the requirement of surface vimentin for viral entry of SARS-CoV and other viruses and the abundance of exposed surface vimentin in senescent cells, it is prudent to hypothesize that cellular senescence plays an essential role in SARS-CoV-2 infection (Fig. 1A). It is yet unknown whether the exposed cell surface 
vimentin interacts with the receptor-binding domain or the fusion core helices. It is also not known clearly which domain of vimentin is utilized.

A

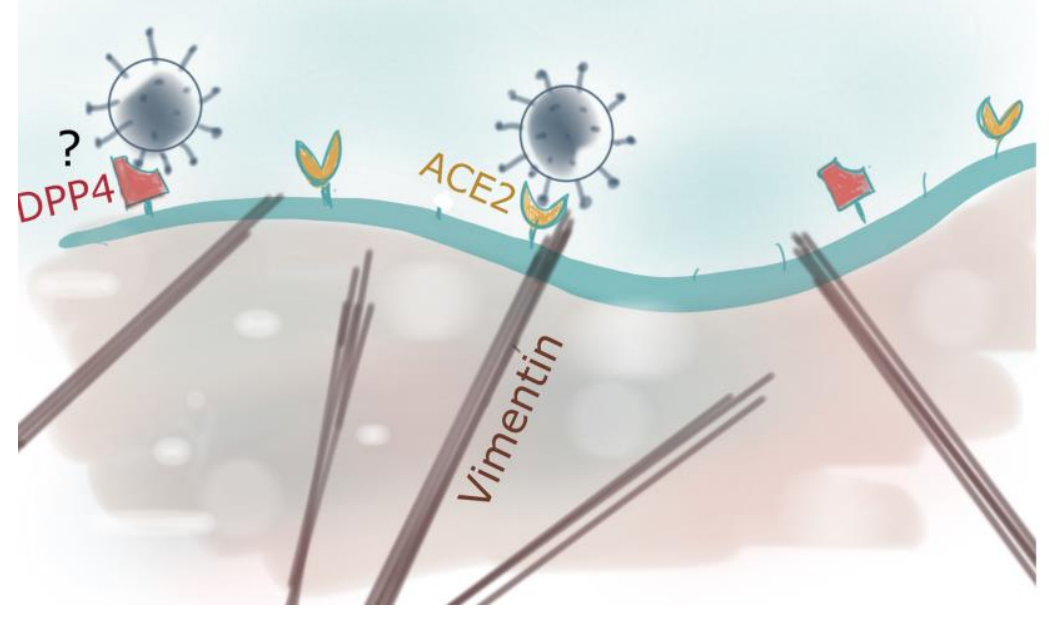

B SARS-COV-2

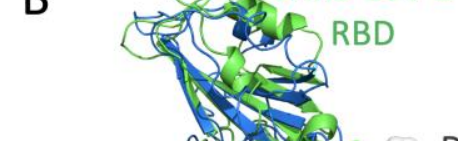

MERS-COV RBD

(i)

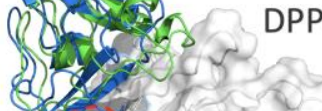

DPP4

Figure 1. A) Initiation of SARS-CoV-2 entry by latching onto ACE2. Surface exposed oxidized form of vimentin in senescence cells may play a role in the viral entry ${ }^{39}$ (or stabilization at the surface). It is not known if and how DPP4 affects the viral entry. B) SARS-CoV-2 RBD overlapped with the MERS-CoV RBD complexed with DPP4 shows a significant steric clash with DPP4, suggesting that binding with DPP4 may only occur via a conformational rearrangement of the RBD region.

MERS-CoV spike protein binds with DPP4, a key receptor that is abundantly expressed in senescent cells

In an attempt to find novel senescent cell surface markers on the plasma-membrane, a recent study used Mass Spectrometry to survey membrane- and cell surface-associated proteins and found that Dipeptidyl peptidase 4 (DPP4) is the most abundant membrane-bound protein localized on the plasma-membrane surface ${ }^{42}$. In fact, normal proliferating cells showed senescence upon overexpression of DPP4, confirmed by the elevated levels of senescence markers p16 and p21, and decrease in anti-senescent marker SIRT1. Importantly, DPP4 is involved in the regulation of glucose level that results in the reduction in insulin secretion, abnormal metabolism of visceral adipose tissues, and the increase in inflammation in type 2 diabetes. 
Human DPP4 is a known host-cell receptor for the spike protein of Middle East respiratory syndrome coronavirus (MERS-CoV) ${ }^{43,44}$. DPP4 mediated immune response dysfunction is implicated in the higher rate of mortality and complications for type 2 diabetes infected with MERS-CoV. Although the effects of DPP4 inhibition is still not clearly understood, DPP4 is considered a potential therapeutic and vaccine target for type 2 diabetes ${ }^{45,46}$. While SARS-CoV is closely related to MERS-CoV, SARS-CoV and SARS-CoV-2 primarily attach to ACE2 instead of DPP4. It is not yet known whether DPP4 is SARS-CoV-2 However, given the high and disproportionate incidence of COVID-19 complications among people with diabetes, it is likely that DPP4 plays a role in SARS-CoV-2 ${ }^{47}$. The structure comparison of the SARS-CoV-2 RBD with the MERS-CoV RBD complexed with DPP4 shows that SARS-CoV-2 structural overlap with DPP4 (Fig. 1B). However, a conformational rearrangement in the RBD overlapping region may still allow binding with DPP4. Consequently, a clinical trial is underway to study the effects of DPP4 inhibitor Linagliptin on type-2 diabetic individuals infected with COVID- $19^{48}$.

In addition to these receptors, other human coronaviruses, including those yet unknown to us, may utilize different receptors for attaching to the human host cells. Besides viral entry, efficient use of host cell machinery is critical for the virus to replicate and egress, and senescence may play a role during any stage of the viral life-cycle. For example, overproduction of vimentin leads to cellular senescence ${ }^{49}$, and cellular vimentin is found to regulate replication and virion assembly of myriads of viruses including the Dengue virus, Influenza A virus ${ }^{50}, \mathrm{HIV}{ }^{51}$ and Human Enterovirus Group B virus ${ }^{52}$, and Bluetongue virus ${ }^{53}$.

\section{Senescence in the respiratory system}

Cellular senescence has increasingly been implicated in aging lungs as well as lungs with conditions such as chronic obstructive pulmonary diseases (COPD), idiopathic pulmonary fibrosis (IPF), and asthma ${ }^{54,55}$. Bronchial and alveolar epithelial cells can develop senescence due to a number of assaults such as smoking, microbial infections, and pollution. Although development of senescence is part of the lung repair mechanism, continuous exposure to the stress factors can result in excessive cellular senescence causing several chronic conditions ${ }^{56,57}$. As such, cigarette smoking is known to cause epithelial inflammation with increased p16 activities in the airways with increased p16 activity and is a major causal factor in COPD pathologies ${ }^{58}$. 


\section{Further outlook}

In summary, this brief review aims to explore the potential role cellular senescence plays in the disparity observed in COVID-19 infection. Mortality. aging, as well as various conditions such as obesity, diabetes, and hypertension, are intricately related to cellular senescence, and individuals with any of these conditions are at increased risk of COVID-19 complications. Molecular mechanisms of the viral entry point to the utilization of key receptors that are also markers of senescent cells. The high correlation of the COVID-19 disparity to cellular senescence makes a compelling case to explore it further in this light. Establishing the connection may allow us to explain the disease pathobiology. For example, senescent cells can harbor virus particles that remain infectious for a long time, resulting in longer incubation times, as happens with HPV-16 virions that remain infectious for 2 weeks on senescent cells ${ }^{4}$. This may also help explain the persistence of the virus for months after infection. The relevance of coronavirus infections with senescence will also allow us to target cellular senescence by therapeutics or by boosting immune recognition of senescent cells ${ }^{3,7,59}$. Ultimately, understanding the molecular mechanisms of how SARS-CoV-2 differentially infects individuals will allow targeted control and combinationtherapeutic strategies to fight off the infection and save lives.

\section{References}

1 Jordan, R. E., Adab, P. \& Cheng, K. K. Covid-19: risk factors for severe disease and death. BMJ 368, m1198, doi:10.1136/bmj.m1198 (2020).

2 Garg, S. et al. Hospitalization Rates and Characteristics of Patients Hospitalized with Laboratory-Confirmed Coronavirus Disease 2019 - COVID-NET, 14 States, March 1-30, 2020. MMWR Morb Mortal Wkly Rep 69, 458-464, doi:10.15585/mmwr.mm6915e3 (2020).

3 Malavolta, M., Giacconi, R., Brunetti, D., Provinciali, M. \& Maggi, F. Exploring the Relevance of Senotherapeutics for the Current SARS-CoV-2 Emergency and Similar Future Global Health Threats. Cells 9, doi:10.3390/cells9040909 (2020).

4 Broniarczyk, J., Ring, N., Massimi, P., Giacca, M. \& Banks, L. HPV-16 virions can remain infectious for 2 weeks on senescent cells but require cell cycle re-activation to allow virus entry. Sci Rep 8, 811, doi:10.1038/s41598-017-18809-6 (2018).

5 He, S. \& Sharpless, N. E. Senescence in Health and Disease. Cell 169, 1000-1011, doi:10.1016/j.cell.2017.05.015 (2017).

6 Campisi, J. Aging, cellular senescence, and cancer. Annu Rev Physiol 75, 685-705, doi:10.1146/annurev-physiol-030212-183653 (2013). 
7 Childs, B. G., Durik, M., Baker, D. J. \& van Deursen, J. M. Cellular senescence in aging and age-related disease: from mechanisms to therapy. Nat Med 21, 1424-1435, doi:10.1038/nm.4000 (2015).

8 Sharpless, N. E. \& Sherr, C. J. Forging a signature of in vivo senescence. Nat Rev Cancer 15, 397-408, doi:10.1038/nrc3960 (2015).

9 Palmer, A. K., Gustafson, B., Kirkland, J. L. \& Smith, U. Cellular senescence: at the nexus between ageing and diabetes. Diabetologia 62, 1835-1841, doi:10.1007/s00125-0194934-x (2019).

10 Carracedo, J. et al. Mechanisms of Cardiovascular Disorders in Patients With Chronic Kidney Disease: A Process Related to Accelerated Senescence. Front Cell Dev Biol 8, 185, doi:10.3389/fcell.2020.00185 (2020).

11 Chilosi, M., Carloni, A., Rossi, A. \& Poletti, V. Premature lung aging and cellular senescence in the pathogenesis of idiopathic pulmonary fibrosis and COPD/emphysema. Transl Res 162, 156-173, doi:10.1016/j.trsl.2013.06.004 (2013).

12 Dodig, S., Cepelak, I. \& Pavic, I. Hallmarks of senescence and aging. Biochem Med (Zagreb) 29, 030501, doi:10.11613/BM.2019.030501 (2019).

13 Kirkland, J. L., Hollenberg, C. H. \& Gillon, W. S. Age, anatomic site, and the replication and differentiation of adipocyte precursors. Am J Physiol 258, C206-210, doi:10.1152/ajpcell.1990.258.2.C206 (1990).

14 Tchkonia, T. et al. Fat tissue, aging, and cellular senescence. Aging Cell 9, 667-684, doi:10.1111/j.1474-9726.2010.00608.x (2010).

15 Franceschi, C. Healthy ageing in 2016: Obesity in geroscience - is cellular senescence the culprit? Nat Rev Endocrinol 13, 76-78, doi:10.1038/nrendo.2016.213 (2017).

16 Minamino, T. et al. A crucial role for adipose tissue p53 in the regulation of insulin resistance. Nat Med 15, 1082-1087, doi:10.1038/nm.2014 (2009).

17 Westhoff, J. H. et al. Hypertension induces somatic cellular senescence in rats and humans by induction of cell cycle inhibitor p16INK4a. Hypertension 52, 123-129, doi:10.1161/HYPERTENSIONAHA.107.099432 (2008).

18 Bernadotte, A., Mikhelson, V. M. \& Spivak, I. M. Markers of cellular senescence. Telomere shortening as a marker of cellular senescence. Aging (Albany NY) 8, 3-11, doi:10.18632/aging.100871 (2016).

19 Rewak, M. et al. Race-related health disparities and biological aging: does rate of telomere shortening differ across blacks and whites? Biol Psychol 99, 92-99, doi:10.1016/j.biopsycho.2014.03.007 (2014).

20 Gardner, M. et al. Gender and telomere length: systematic review and meta-analysis. Exp Gerontol 51, 15-27, doi:10.1016/j.exger.2013.12.004 (2014).

21 Burton, D. G. A. \& Stolzing, A. Cellular senescence: Immunosurveillance and future immunotherapy. Ageing Res Rev 43, 17-25, doi:10.1016/j.arr.2018.02.001 (2018).

22 Sagiv, A. et al. NKG2D ligands mediate immunosurveillance of senescent cells. Aging (Albany NY) 8, 328-344, doi:10.18632/aging.100897 (2016).

23 Zingoni, A. et al. NKG2D and Its Ligands: "One for All, All for One". Front Immunol 9, 476, doi:10.3389/fimmu.2018.00476 (2018). 
24 Soto-Gamez, A., Quax, W. J. \& Demaria, M. Regulation of Survival Networks in Senescent Cells: From Mechanisms to Interventions. J Mol Biol 431, 2629-2643, doi:10.1016/j.jmb.2019.05.036 (2019).

25 Coppe, J. P. et al. Senescence-associated secretory phenotypes reveal cellnonautonomous functions of oncogenic RAS and the p53 tumor suppressor. PLoS Biol 6, 2853-2868, doi:10.1371/journal.pbio.0060301 (2008).

26 Kang, T. W. et al. Senescence surveillance of pre-malignant hepatocytes limits liver cancer development. Nature 479, 547-551, doi:10.1038/nature10599 (2011).

27 van Tuyn, J. et al. Oncogene-Expressing Senescent Melanocytes Up-Regulate MHC Class II, a Candidate Melanoma Suppressor Function. J Invest Dermatol 137, 2197-2207, doi:10.1016/j.jid.2017.05.030 (2017).

28 Frescas, D. et al. Senescent cells expose and secrete an oxidized form of membranebound vimentin as revealed by a natural polyreactive antibody. Proc Natl Acad Sci U S A 114, E1668-E1677, doi:10.1073/pnas.1614661114 (2017).

29 Baz-Martinez, M. et al. Cell senescence is an antiviral defense mechanism. Sci Rep 6, 37007, doi:10.1038/srep37007 (2016).

30 Antonangeli, F., Zingoni, A., Soriani, A. \& Santoni, A. Senescent cells: Living or dying is a matter of NK cells. J Leukoc Biol 105, 1275-1283, doi:10.1002/JLB.MR0718-299R (2019).

31 Kim, J. A., Seong, R. K. \& Shin, O. S. Enhanced Viral Replication by Cellular Replicative Senescence. Immune Netw 16, 286-295, doi:10.4110/in.2016.16.5.286 (2016).

32 Dianzani, F. Interferon treatments: how to use an endogenous system as a therapeutic agent. J Interferon Res Spec No, 109-118, doi:10.1089/jir.1992.1992.109 (1992).

33 Kuilman, T. et al. Oncogene-induced senescence relayed by an interleukin-dependent inflammatory network. Cell 133, 1019-1031, doi:10.1016/j.cell.2008.03.039 (2008).

34 Raulet, D. H. \& Guerra, N. Oncogenic stress sensed by the immune system: role of natural killer cell receptors. Nat Rev Immuno/ 9, 568-580, doi:10.1038/nri2604 (2009).

35 Oishi, N. et al. Hepatitis B virus X protein overcomes oncogenic RAS-induced senescence in human immortalized cells. Cancer Sci 98, 1540-1548, doi:10.1111/j.13497006.2007.00579.x (2007).

36 Beachy, T. M., Cole, S. L., Cavender, J. F. \& Tevethia, M. J. Regions and activities of simian virus $40 \mathrm{~T}$ antigen that cooperate with an activated ras oncogene in transforming primary rat embryo fibroblasts. J Virol 76, 3145-3157, doi:10.1128/jvi.76.7.31453157.2002 (2002).

37 Schutze, D. M. et al. Differential in vitro immortalization capacity of eleven (probable) [corrected] high-risk human papillomavirus types. J Viro/ 88, 1714-1724, doi:10.1128/JVI.02859-13 (2014).

38 Jeffers, S. A. et al. CD209L (L-SIGN) is a receptor for severe acute respiratory syndrome coronavirus. Proc Natl Acad Sci U S A 101, 15748-15753, doi:10.1073/pnas.0403812101 (2004).

$39 \mathrm{Yu}, \mathrm{Y}$. T. et al. Surface vimentin is critical for the cell entry of SARS-CoV. J Biomed Sci 23, 14, doi:10.1186/s12929-016-0234-7 (2016).

$40 \mathrm{Du}, \mathrm{N}$. et al. Cell surface vimentin is an attachment receptor for enterovirus 71. J Virol 88, 5816-5833, doi:10.1128/JVI.03826-13 (2014). 
41 Koudelka, K. J. et al. Endothelial targeting of cowpea mosaic virus (CPMV) via surface vimentin. PLoS Pathog 5, e1000417, doi:10.1371/journal.ppat.1000417 (2009).

$42 \mathrm{Kim}, \mathrm{K}$. M. et al. Identification of senescent cell surface targetable protein DPP4. Genes Dev 31, 1529-1534, doi:10.1101/gad.302570.117 (2017).

43 Lu, G. et al. Molecular basis of binding between novel human coronavirus MERS-CoV and its receptor CD26. Nature 500, 227-231, doi:10.1038/nature12328 (2013).

44 Wang, N. et al. Structure of MERS-CoV spike receptor-binding domain complexed with human receptor DPP4. Cell Res 23, 986-993, doi:10.1038/cr.2013.92 (2013).

45 Pang, Z. et al. Therapeutic vaccine against DPP4 improves glucose metabolism in mice. Proc Natl Acad Sci U S A 111, E1256-1263, doi:10.1073/pnas.1322009111 (2014).

46 Gallwitz, B. Clinical Use of DPP-4 Inhibitors. Front Endocrinol (Lausanne) 10, 389, doi:10.3389/fendo.2019.00389 (2019).

47 lacobellis, G. COVID-19 and diabetes: Can DPP4 inhibition play a role? Diabetes Res Clin Pract 162, 108125, doi:10.1016/j.diabres.2020.108125 (2020).

48 lacobellis, G. Effects of DPP4 Inhibition on COVID-19. (2020).

49 Nishio, K., Inoue, A., Qiao, S., Kondo, H. \& Mimura, A. Senescence and cytoskeleton: overproduction of vimentin induces senescent-like morphology in human fibroblasts. Histochem Cell Biol 116, 321-327, doi:10.1007/s004180100325 (2001).

50 Wu, W. et al. RIG-I and TLR3 are both required for maximum interferon induction by influenza virus in human lung alveolar epithelial cells. Virology 482, 181-188, doi:10.1016/j.virol.2015.03.048 (2015).

51 Fernandez-Ortega, C. et al. Identification of Vimentin as a Potential Therapeutic Target against HIV Infection. Viruses 8, doi:10.3390/v8060098 (2016).

52 Turkki, P., Laajala, M., Flodstrom-Tullberg, M. \& Marjomaki, V. Human Enterovirus Group B Viruses Rely on Vimentin Dynamics for Efficient Processing of Viral Nonstructural Proteins. J Virol 94, doi:10.1128/JVI.01393-19 (2020).

53 Bhattacharya, B., Noad, R. J. \& Roy, P. Interaction between Bluetongue virus outer capsid protein VP2 and vimentin is necessary for virus egress. Virol J 4, 7, doi:10.1186/1743-422X-4-7 (2007).

54 Bartling, B. Cellular senescence in normal and premature lung aging. $Z$ Gerontol Geriatr 46, 613-622, doi:10.1007/s00391-013-0543-3 (2013).

55 Hadj Salem, I., Dube, J., Boulet, L. P. \& Chakir, J. Telomere shortening correlates with accelerated replicative senescence of bronchial fibroblasts in asthma. Clin Exp Allergy 45, 1713-1715, doi:10.1111/cea.12611 (2015).

56 Nyunoya, T. et al. Cigarette smoke induces cellular senescence. Am J Respir Cell Mol Biol 35, 681-688, doi:10.1165/rcmb.2006-01690C (2006).

57 Kheradmand, F., You, R., Hee Gu, B. \& Corry, D. B. Cigarette Smoke and DNA Cleavage Promote Lung Inflammation and Emphysema. Trans Am Clin Climatol Assoc 128, $222-$ 233 (2017).

58 Cottage, C. T. et al. Targeting p16-induced senescence prevents cigarette smokeinduced emphysema by promoting IGF1/Akt1 signaling in mice. Commun Biol 2, 307, doi:10.1038/s42003-019-0532-1 (2019).

59 Pereira, B. I. et al. Senescent cells evade immune clearance via HLA-E-mediated NK and CD8(+) T cell inhibition. Nat Commun 10, 2387, doi:10.1038/s41467-019-10335-5 (2019). 
